\title{
An investigation of the convergence to the stationary state in the Hassell mapping
}

\author{
Hans M.J. de Mendonça ${ }^{a, *}$, Edson D. Leonel ${ }^{\mathrm{a}, \mathrm{b}}$, Juliano A. de Oliveira ${ }^{\mathrm{a}, \mathrm{c}}$ \\ a Departamento de Física, UNESP - Univ Estadual Paulista, Av. 24A, 1515, Bela Vista, 13506-900, Rio Claro, SP, Brazil \\ ${ }^{\mathrm{b}}$ Abdus Salam International Center for Theoretical Physics, Strada Costiera 11, 34151 Trieste, Italy \\ ${ }^{\mathrm{c}}$ UNESP - Univ Estadual Paulista - Câmpus de São João da Boa Vista, Av. Professora Isette Corrêa Fontão, 505 - Jardim Santa Rita das \\ Areias, 13876-750 - São João da Boa Vista, SP, Brazil
}

\section{A R T I C L E I N F O}

Article history:

Received 20 September 2016

Available online 28 September 2016

\section{Keywords:}

Hassell map

Bifurcations

Scaling law

\begin{abstract}
A B S T R A C T
We investigate the convergence to the fixed point and near it in a transcritical bifurcation observed in a Hassell mapping. We considered a phenomenological description which was reinforced by a theoretical description. At the bifurcation, we confirm the convergence for the fixed point is characterized by a homogeneous function with three exponents. Near the bifurcation the decay to the fixed point is exponential with a relaxation time given by a power law. Although the expression of the mapping is different from the traditional logistic mapping, at the bifurcation and near it, the local dynamics is essentially the same for either mappings.
\end{abstract}

(C) 2016 Elsevier B.V. All rights reserved.

\section{Introduction}

The study of the properties for nonlinear systems has been of interest of many authors along the decades. Sometimes these properties are associated with bifurcations cascades of dynamical systems that describe mathematically natural phenomena [1-3]. The interest for the subject increased since the work of May [4], which has applications to biology [5]. Since then, many different applications involving mappings were considered in different areas including physics [6-8], mathematics, biology, chemistry, engineering among others [9-20].

In this paper, we consider the Hassell mapping [21-23] with the main goal of understanding and describing the behavior of the convergence to the fixed point at the bifurcation and near it. We concentrate particularly on the transcritical bifurcation where two coexisting fixed points exchange their stability. We confirm that at the bifurcation the convergence to the fixed point is described by a homogeneous function leading to three scaling exponents. Near the bifurcation the convergence is no longer described by a homogeneous function but instead it is given by an exponential decay. The relaxation time is confirmed to be described by a power law of the distance of the bifurcation with a slope -1 .

This paper is organized as follows. In Section 2 the mapping, the equilibrium conditions and a phenomenological approach leading to a scaling law is described. Then in Section 3 a mathematical investigation is made by transforming the difference equation into a differential equation leading to an easier solution. Such approach gives the exponents obtained in Section 2 analytically. Our conclusion are made in Section 4.

\footnotetext{
* Corresponding author.

E-mail addresses: hans.mendonca@yahoo.com.br (H.M.J. de Mendonça), julianoantonio@sjbv.unesp.br (J.A. de Oliveira).
} 


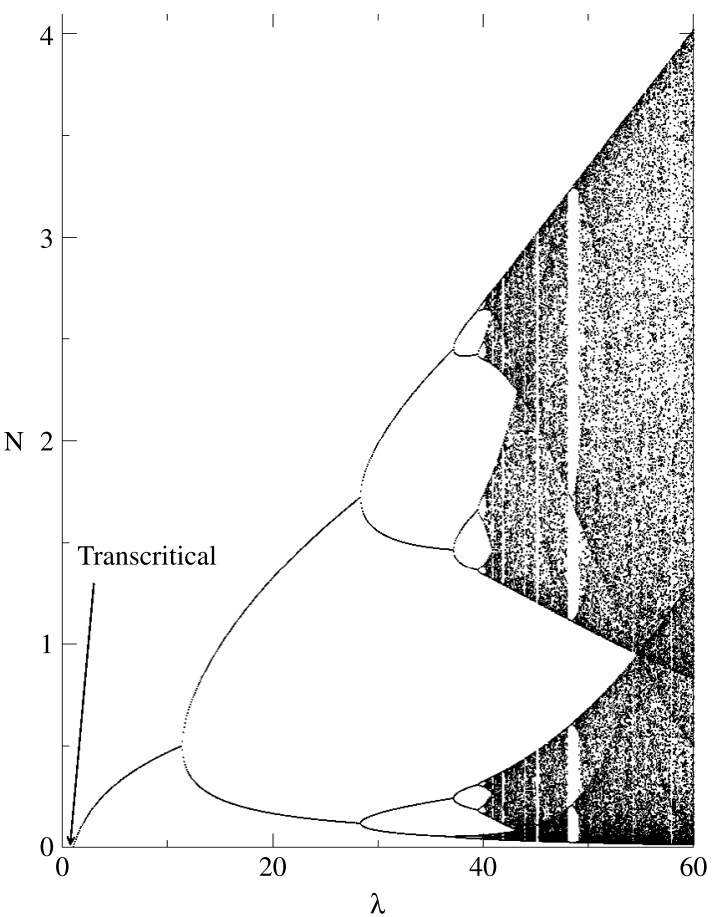

Fig. 1. Bifurcation diagram obtained for Eq. (1) using $\gamma=6$ and $a=1$. Transcritical bifurcation is shown in the figure.

\section{The model and scaling properties}

The model we consider is the Hassell mapping written as [21-23]

$$
N_{n+1}=\frac{\lambda N_{n}}{\left(1+a N_{n}\right)^{\gamma}},
$$

where $\lambda, a$ and $\gamma$ are control parameters and $N$ is the dynamical variable. For the case of $\gamma=1$ the Beverton-Holt model [22] is recovered. Fig. 1 shows the bifurcation diagram for $\gamma=6$ and $a=1$. We notice that for $\lambda=1$ a transcritical bifurcation occurs. The fixed points are obtained using the condition $N_{n+1}=N_{n}=N^{*}$, which leads to the existence of two fixed points. One is $N^{*}=0$, which is stable (asymptotically stable) for $\lambda \in[0,1)$ while the other one is $N^{*}=\left(\lambda^{1 / \gamma}-1\right) / a$, stable for the range $\lambda \in(1,11.3)$. After this limit a sequence of period doubling bifurcation is observed leading to chaos. Periodic windows are also observed for large values of $\lambda$.

Our main goal is to investigate the convergence to the fixed point $N^{*}=0$ at the transcritical bifurcation at $\lambda_{c}=1$ and near its neighboring such that $\mu=\frac{\lambda_{c}-\lambda}{\lambda_{c}} \ll 1$, with $\lambda \leq \lambda_{c}$. To do that, the most natural variable to be considered in the investigation is the deviation from the fixed point [24]. For $N^{*}=0$, the distance for the fixed point is represented by the dynamical variable $N$. Certainly the convergence to the steady state depends also on the number of iterations $n$, on the initial condition $N_{0}$, and on the parameter $\mu=\frac{\lambda_{c}-\lambda}{\lambda_{c}}$. The quantity $\mu=0$ defines the bifurcation point. For such a parameter the convergence to the fixed point is shown in Fig. 2 for $a=1, \gamma=6$ and different initial conditions $N_{0}$, as labeled in the figure.

From Fig. 2 we see that depending on the initial condition $N_{0}$, the orbit keeps itself confined in a plateau of constant $N$. Later on after reaching a crossover iteration number, $n_{x}$, the orbit experiences a changeover from a constant regime progressing to a power law decay described by an exponent $\beta$. The size of the plateau depends on the initial $N_{0}$. From Fig. 2 we propose that: (i) For short $n$, say $n \ll n_{x}$, the behavior of $N v s$. $n$ is given by $N(n) \propto N_{0}^{\alpha}$ and, because $N \propto N_{0}$, we conclude $\alpha=1$; (ii) For $n \gg n_{x}$, we observed $N(n) \propto n^{\beta}$ where $\beta$ is called a decay exponent; (iii) The crossover iteration number $n_{x}$ is given by $n_{x} \propto N_{0}^{z}$ where $z$ is a changeover exponent.

The exponent $\beta$ can be obtained from fitting a power law to the decay regime. From Fig. 2 we obtained $\beta=$ $-0.99999960(1) \simeq-1$ considering $\gamma=6$ and $a=1$. The exponent $z$ is obtained from the plot $n_{x} v s$. $N_{0}$, where $n_{x}$ is obtained as the crossing of the constant plateau by the power law decay, as shown in Fig. 3. The slope obtained for $\gamma=6$ and $a=1$, as shown in Fig. 3 is $z=-1$. Several other values of $\gamma$ were considered and the values of $\beta$ and $z$ are independent of $\gamma$.

The three scaling suppositions allow us to describe the behavior of $N$ as a homogeneous function of the variables $n$ and $N_{0}$ of the type

$$
N\left(N_{0}, n\right)=\operatorname{lN}\left(l^{b} N_{0}, l^{c} n\right),
$$




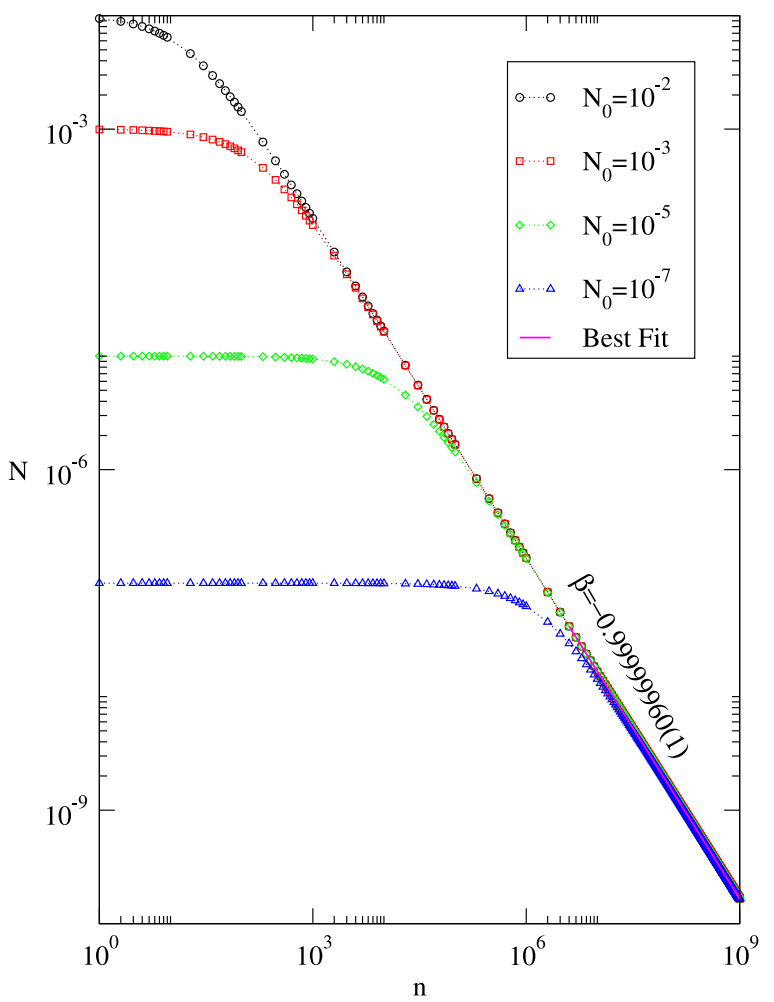

Fig. 2. Convergence to the fixed point at $N^{*}=0$ for the parameters $\gamma=6, a=1$ and different initial conditions as labeled in the figure. At the bifurcation $\mu=0$.

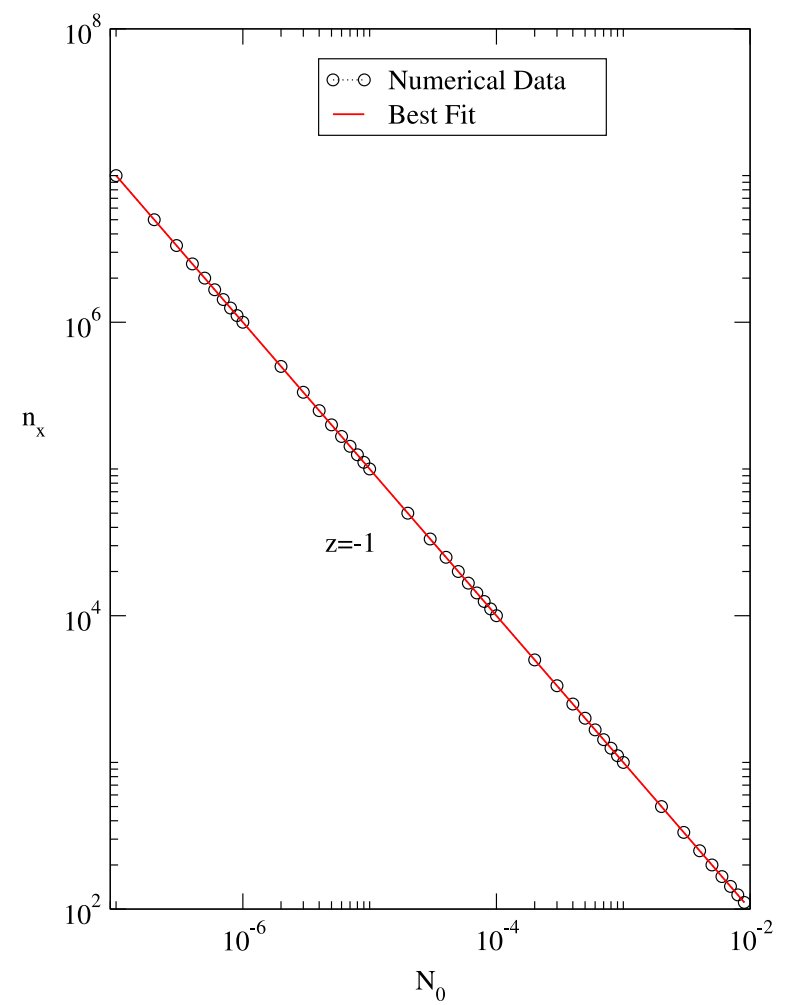

Fig. 3. Plot of the crossover iteration number $n_{x}$ against the initial condition $x_{0}$ together with their power law fitting for $\gamma=6$ and 1 with slope $z=-1$. 


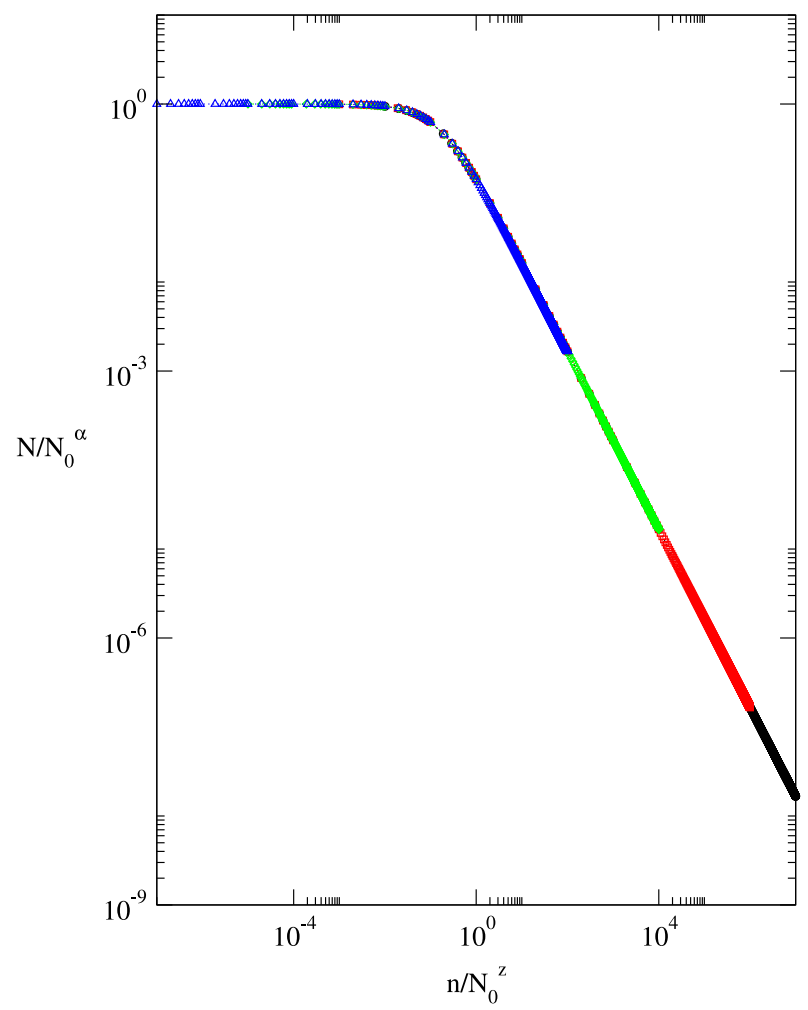

Fig. 4. Overlap of all curves shown in Fig. 2 onto a single and universal plot, after a convenient rescale of the axis.

where $l$ is a scaling factor, $b$ and $c$ are characteristic exponents. Using a similar procedure made in Refs. [25,26], we end up with the following scaling law

$$
z=\frac{\alpha}{\beta} \text {. }
$$

The knowledge of any two exponents allow to find the third one by using Eq. (3). The exponents are also used to rescale the variables $N$ and $n$ such that $N \rightarrow N / N_{0}^{\alpha}$ and $n \rightarrow n / N_{0}^{z}$ and overlap all curves of $N v s$. $n$ into a universal plot as shown in Fig. 4.

Let us discuss now the dynamics of $N(n)$ for $\mu \neq 0$. This characterizes the neighboring of the bifurcation. The evolution to the fixed point is given by an exponential law written as $[24,27]$

$$
N(n, \mu) \propto \mathrm{e}^{-n / \tau},
$$

where $\tau$ is the relaxation time and has the following form

$$
\tau \propto \mu^{\delta}
$$

and $\delta$ is a relaxation exponent. Fig. 5 shows the behavior of $\tau$ vs. $\mu$. We plot only the curve obtained for $\gamma=6$ although other values of $\gamma$ yield the same result. A power law fitting furnishes the exponent $\delta \cong-1$ and is independent on the value of the parameter $\gamma$.

\section{A theoretical approach to the fixed point}

To obtain the exponents discussed in Section 2 in a more rigorous way we consider two cases: (i) at the bifurcation point $\lambda=\lambda_{c}=1$, setting $\mu=0$; and (ii) near the bifurcation for $\lambda \neq \lambda_{c}$, yielding $\mu \neq 0$. We start first with case (i). Expanding the mapping (1) in Taylor's series of $N_{n}$ when $a N_{n} \ll 1$ we obtain

$$
N_{n+1} \simeq \lambda N_{n}\left[1-\gamma a N_{n}-\frac{1}{2} \gamma a^{2}(-\gamma-1) N_{n}^{2}-O(3)\right] .
$$

Near the fixed point, $N$ is close to 0 , then we can keep only low orders in the expansion. Considering the terms of the first order, we obtain

$$
N_{n+1}=\lambda N_{n}\left(1-\gamma a N_{n}\right) .
$$




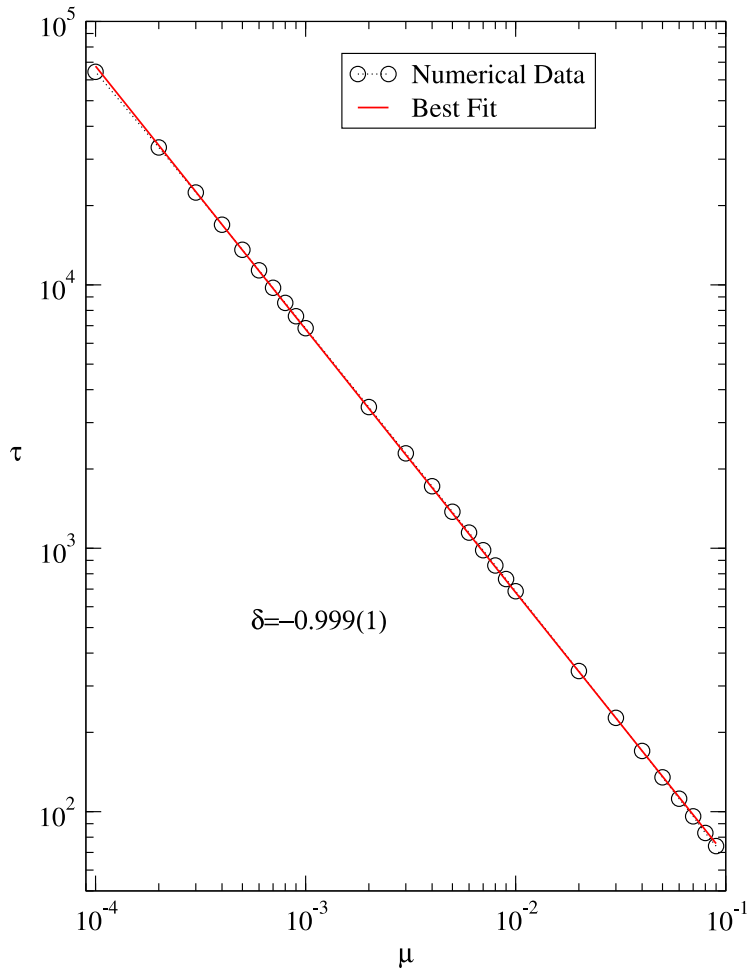

Fig. 5. Plot of the relaxation to the fixed point as a function of $\mu$ for the control parameters $\gamma=6$ and $a=1$.

Under this condition, we suppose the dynamical variable $N$ can be considered as a continuous variable. Hence Eq. (7) is rewritten as

$$
\begin{aligned}
N_{n+1}-N_{n} & =\frac{N_{n+1}-N_{n}}{(n+1)-n}, \\
& \approx \frac{\mathrm{d} N}{\mathrm{~d} n}=-\gamma a N^{2} .
\end{aligned}
$$

It is important to mention that a similar approach was used with success in a conservative system as discussed in Ref. [28].

Grouping the terms properly we obtain the following differential equation

$$
\frac{\mathrm{d} N}{N^{2}}=-\gamma a \mathrm{~d} n \text {. }
$$

The initial condition $N_{0}$ is defined for $n=0$ while for an arbitrary $n$ we have $N(n)$. Using these variables as limits of the integrals we have

$$
\int_{N_{0}}^{N(n)} \frac{\mathrm{d} N^{\prime}}{N^{\prime 2}}=-\int_{0}^{n} \gamma a \mathrm{~d} n^{\prime}
$$

After integration we obtain

$$
N(n)=\frac{1}{\left[\gamma a n+\frac{1}{N_{0}}\right]} .
$$

Let us then discuss the implications of Eq. (11) for specific ranges of $n$. We start with the case $\gamma a n \ll 1 / N_{0}$, which is equivalent to the previous section of $n \ll n_{x}$. For such a case we obtain that $N(n) \cong N_{0}$. A comparison with first scaling hypothesis allows us to conclude that $a=1$. Second we consider the situation $\gamma$ an $\gg 1 / N_{0}$, corresponding to $n \gg n_{x}$ in the previous section. For such case we obtain that

$$
N(n) \approx n^{-1} .
$$

Comparing then this result with the second scaling hypothesis we conclude $\beta=-1$. Finally when $\gamma$ an $=1 / N_{0}$, which is the case of $n=n_{x}$, we obtain

$$
n_{x} \cong N_{0}^{-1} \text {. }
$$


A comparison with third scaling hypothesis gives $z=-1$. Using this procedure we obtained all the three exponents discussed in the previous section.

When $\mu \neq 0$ we can rewrite the mapping as

$$
\begin{aligned}
N_{n+1}-N_{n} & =\lambda N_{n}\left(1-a N_{n}\right)-N_{n}, \\
& =\frac{N_{n+1}-N_{n}}{(n+1)-n} \approx \frac{\mathrm{d} N}{\mathrm{~d} n}, \\
& =-(1-\lambda) N-\lambda a N^{2} .
\end{aligned}
$$

However, near the fixed point $N \cong 0$ consider the term $N^{2}$ going to zero faster than $N$. Thus, the last term of Eq. (14) is neglected and we have

$$
\frac{\mathrm{d} N}{\mathrm{~d} n}=-\mu N
$$

where $\mu=1-\lambda$. Considering again that for $n=0$ the initial condition is $N_{0}$, we have to integrate the following equation

$$
\int_{N_{0}}^{N(n)} \frac{\mathrm{d} N^{\prime}}{N^{\prime}}=-\mu \int_{0}^{n} \mathrm{~d} n^{\prime}
$$

After integration and grouping the terms we obtain

$$
N(n)=N_{0} \mathrm{e}^{-\mu n} .
$$

Comparing this result with Eqs. (4) and (5) we conclude that the exponent $\delta=-1$ is in well agreement with Fig. 5 . Moreover, for the Hassell model the numerical values of the exponents $\alpha, \beta, z$ and $\delta$ are independent of $\gamma$. The exponents are indeed the same as the ones observed in the traditional logistic map.

\section{Discussions and conclusions}

We have considered the convergence to the fixed point at the transcritical bifurcation in the Hassell mapping. At the bifurcation point we used a phenomenological description and prove that the decay to the fixed point is characterized by a homogeneous function with three exponents. They are related to each other by a scaling law $z=\alpha / \beta$. Our results gave $\alpha=1, \beta=-1, z=-1$. Near the bifurcation the convergence to the fixed point is marked by an exponential decay and the relaxation time is described by a power law of the type $\tau \propto \mu^{\delta}$ with $\delta=-1$. The exponents obtained are independent of the parameter $\gamma$. The four exponents obtained here allow us to conclude that near the transcritical bifurcation the Hassell map is behaving essentially as a logistic map and Eq. (7) is a confirmation of that.

Further investigation was also made at the period doubling bifurcation happening at $\lambda=11.39062500 \ldots$ for $\gamma=6$ and $a=1$. The exponents obtained were $\alpha=1, \beta=-1 / 2, z=-2$ and $\delta=-1$ in well agreement with results discussed in Ref. [26].

\section{Acknowledgments}

HMJM thanks FAPESP (2015/22062-3), JAO thanks PROPe/UNESP, CNPq (311105/2015-7) and FAPESP (2014/18672-8) for financial support and EDL thanks to CNPq (303707/2015-1), FUNDUNESP and FAPESP (2012/23688-5) (Brazilian agencies).

\section{References}

[1] A.J. Lichtenberg, M.A. Lieberman, Regular and Chaotic Dynamics, in: Appl. Math. Sci., vol. 38, Springer Verlag, NY, 1992.

[2] A. Palacios, Internat. J. Bifur. Chaos 12 (2002) 1859.

[3] P.G. Lind, J. Corte-Real, J.A.C. Gallas, Physica A 327 (2003) 65.

[4] R.M. May, Science 86 (1974) 645.

[5] R.M. May, G.A. Oster, Am. Nat. 110 (1976) 573.

[6] C. Grebogi, E. Ott, J.A. Yorke, Phys. Rev. Lett. 48 (1982) 1507; Physica D 7 (1983) 181.

[7] J.R. Pounder, T.D. Rogers, Nonlinear Anal. TMA 10 (1986) 415.

[8] M. Joglekar, E. Ott, A. Yorke, Phys. Rev. Lett. 113 (2014) 084101

[9] K. Hamacher, Chaos 22 (2012) 033149.

[10] M. McCartney, Chaos 21 (2012) 043104.

[11] P. Philominathan, M. Santhiah, I.R. Mohamed, et al., Internat. J. Bifur. Chaos 21 (2011) 1927.

[12] M. Santhiah, P. Philominathan, Pramana J. Phys. 75 (2010) 403.

[13] Y.-G. Zhang, J.-F. Zhang, Q. Ma, et al., Int. J. Nonlinear Sci. Numer. Simul. 11 (2010) 157.

[14] H. Wen, Z. Guang-Hao, Z. Gong, et al., Acta Phys. Sin. 17 (2012) 170505.

[15] M. Urquizu, A.M. Correig, Chaos Solitons Fractals 33 (2007) 1292.

[16] G. Livadiotis, Adv. Complex Syst. 8 (2005) 15.

[17] D. Ilhem, K. Amel, Discrete Dyn. Nat. Soc. 2006 (2006) Article ID 15840.

[18] T.Y. Li, J.A. Yorke, Amer. Math. Monthly 82 (1975) 985.

[19] J.A.C. Gallas, Phys. Rev. Lett. 70 (1983) 2714. 
[20] P. Collet, J.-P. Eckmann, Iterated Maps on the Interval as Dynamical Systems, Birkhauser, Boston, MA, 1980

[21] K. Sakai, Nonlinear Dynamics and Chaos in Agricultural Systems, Elsevier Science, Tokyo, 2001.

[22] M.J. Panik, Growth Curve Modeling: Theory and Applications, John Wiley, Canada, 2014.

[23] J. Bascompte, R.V. Sol, J. Anim. Ecol. 63 (1994) 256.

[24] E.D. Leonel, J.K.L. da Silva, S.O. Kamphorst, Internat. J. Bifur. Chaos 12 (2002) 1667.

[25] R.M.N. Teixeira, D.S. Rando, F.C. Geraldo, R.N. Costa Filho, J.A. de Oliveira, E.D. Leonel, Phys. Lett. A 379 (2015) 1246

[26] E.D. Leonel, R.M.N. Teixeira, D.S. Rando, R.N. Costa Filho, J.A. de Oliveira, Phys. Lett. A 379 (2015) 1796.

[27] P.C. Hohenberg, B.I. Halperin, Rev. Modern Phys. 49 (1977) 435.

[28] G. Corso, F.B. Rizzato, Phys. Rev. E 58 (1998) 8013. 